\title{
Triggering and the gravitational instability in shells and supershells
}

\author{
Jan Palouš \\ Astronomical Institute, Academy of Sciences of the Czech Republic, Boční II 1401, \\ 14041 Prague 4, Czech Republic \\ email: palous@ig.cas.cz
}

\begin{abstract}
The gravitational instability of shells and supershells and its relevance to the triggering of the star formation in a turbulent ISM is discussed. The IMF of the self-gravitating clumps formed out of shells is computed and triggering time is compared with the shell dissolution time due to chaotic motions.
\end{abstract}

Keywords. shock waves, turbulence, ISM: bubbles, ISM: clouds, stars: formation

\section{Introduction}

Elmegreen \& Lada (1978) assume that new stars form in the Cold Post-Shock (CPS) layer separating the shock front from the ionisation front when an HII region created with the energy inserted to the interstellar medium by the previous generation of stars propagates into the dense molecular cloud. The early fast expansion slows down to $\sim 5 \mathrm{~km}$ $\mathrm{s}^{-1}$, which eliminates the stabilising effect of stretching. In the CPS, the gravitational instability is triggered when it is dense enough $\left(\geqslant 10^{5} \mathrm{~cm}^{-3}\right)$ at the temperature $\sim 10^{2} \mathrm{~K}$. The gravitational instability of the CPS layer reorganises the accumulated matter forming the clumps, which may be the places of new star formation.

The concept of a turbulent ISM is developed by Elmegreen \& Scalo (2004) and Scalo \& Elmegreen (2004). The self-similar changing structures cannot be interpreted in terms of simple equilibrium quantities such as velocity dispersion or pressure. Interacting supersonic shock waves create temporary structures including clumps with a range of sizes and masses, which form and dissolve in sheets and filaments. The energy dissipation in supersonic shocks and cooling is compensated with a variety of driving mechanisms including the galactic rotation and galaxy interactions on the large scale, and energy inserted by stars on the small scale.

Here, we discuss if the shell triggering may be important to formation of longer-living star clusters and stars in the ISM clouds.

\section{Bubble Bath in the ISM}

Shells and supershells in the Milky Way are analysed by Ehlerová \& Palouš (2005), who discovered more than 600 structures in the Leiden Dwingeloo HI survey. This analysis is extended with the Leiden/Argentine/Bonn survey, see the contribution by Ehlerová (this volume). There is a lot of structures discovered in other nearby galaxies including LMC, SMC, M31 etc. Reviews of the observational evidence are given by Stanimirovic (this volume) and Brinks (this volume).

The static ISM in two or more fixed components does not exist. The shells are frequently seen in expansion. They move relatively to each other, collide and open to the 
galactic halo resembling a bubble bath in a hot spring. The high density, low temperature filaments and sheets have $1 \mathrm{D}$ or $2 \mathrm{D}$ geometry. They form and go showing that the individual structures are far away from an equilibrium situation.

Stars insert energy and mass into this bubble bath. In some cases, we even see the powering $\mathrm{OB}$ association, but in the majority of shells the parent stars are not detected. It shows that the average lifetime of shells is longer compared to the lifetime of young and massive stars. Some of the supershells, e.g. those seen in the LMC and SMC, may be connected to galaxy versus galaxy interactions or to galaxy rotation, spiral arms and bars rather than to the star formation. Other structures are due to cold gas infall coming to the galaxy plane in the form of high velocity clouds, which produce incomplete partially open shell-like features. The impacts of high velocity clouds are more destructive and unable to create spherical-like shells. Another mechanism creating or enlarging the existing structures is stripping with ram pressure created by a galaxy high speed motion through the diluted and hot intracluster medium.

\section{Shell fragmentation: IMF of clumps}

A dispersion relation gives how the frequency $\omega(k)=2 \pi / t_{\text {growth }}$ depends on the mode wave number $k$. Within a volume inside of radius $R$, the number of fragments produced by an instability is given as $N=\omega \frac{R^{3}}{(\lambda / 4)^{3}}=\frac{8 \omega R^{3} k^{3}}{\pi^{3}}, k=\frac{2 \pi}{\lambda}$, where $\lambda$ is the wave length. The mass of a fragment is $m=\frac{4}{3} \pi(\lambda / 4)^{3} \rho=\frac{1}{6} \pi^{4} \rho k^{-3}$, and the number of fragments with the wave number in the interval $(k, k+d k)$ is $d N=\frac{24 \omega R^{3} k^{2}}{\pi^{3}} d k$. The mass spectrum $\xi(m)=d N / d m$, or the number of clumps in an interval of mass, is $\xi(m)=-\frac{4}{3} \pi R^{3} \rho \omega m^{-2}$

The dispersion relation of the Jeans gravitational instability in $3 \mathrm{D}$ is

$$
\omega^{2}(k)=-c^{2} k^{2}+4 \pi G \rho,
$$

where $c$ is the speed of sound. It transforms to the mass spectrum

$$
\xi_{\text {Jeans }}(m)=\frac{16}{9} R^{3} \rho m^{-2}\left[-c^{2}\left(\frac{\pi^{4} \rho}{6 m}\right)^{2 / 3}+4 \pi G \rho\right]^{1 / 2},
$$

showing for large masses the slope $\alpha=-2$. It flattens when the fragment mass decreases to the Jeans mass $m_{\text {Jeans }}=\frac{\pi^{5 / 2}}{48} G^{-3 / 2} c^{3} \rho^{-1 / 2}$.

In thin CPS layer with the $2 \mathrm{D}$ geometry, the dispersion relation is

$$
\omega^{2}(k)=-c_{s h}^{2} k^{2}+2 \pi G \Sigma_{s h} k,
$$

where $c_{s h}$ is the speed of sound inside of the CPS and $\Sigma_{s h}$ is its mass column density. The mass spectrum of CPS fragments is

$$
\xi(m)=\frac{1}{4} A \pi^{3 / 4} \Sigma_{s h}^{3 / 2} m^{-9 / 4} \times \frac{-3 \pi^{1 / 2} c_{s h}^{2} m^{-1 / 2}+2.5 G \Sigma_{s h}^{1 / 2}}{\left(-\pi^{1 / 2} c_{s h}^{2} m^{-1 / 2}+G \Sigma_{s h}^{1 / 2}\right)^{1 / 2}},
$$

where $A$ is the area of the CPS, see Elmegreen \& Elmegreen (1978). At the high mass end is the CPS mass spectrum steeper, compared to 3D Jeans mass spectrum, with $\alpha=-2.25$. The expansion would make it even more steep, with the slope $\alpha=-2.35$, see Palouš et al. (2003). 
Table 1. The time when the gravitational instability sets in a CPS

\begin{tabular}{|l|l|l|l|}
\hline $\begin{array}{l}c_{s h}\left[\mathrm{kms}^{-1}\right] \\
n\left[\mathrm{~cm}^{-3}\right]\end{array}$ & 0.3 & 0.5 & 1.0 \\
\hline $10^{-1}$ & 43 & 59 & 91 \\
1 & 14 & 19 & 29 \\
$10^{2}$ & 1.4 & 1.9 & 2.9 \\
$10^{6}$ & 0.01 & 0.02 & 0.03
\end{tabular}

\section{Velocity - Size Relation from Triggering in CPS Layers}

We may ask when the gravitational instability starts in a thin shell expanding into a dense cloud. This triggering time of the gravitational instability of the CPS is given in Table 1 for different densities of the clouds $n$ and for three values of $c_{s h}$.

The triggering time given in Table 1 sets a velocity - size relation as it is shown in Fig. 1. Having a cloud with expanding shells, it shows an expansion velocity as a function of size under the condition that shells are able to trigger the gravitational instability. When, for given size $\Delta r$, is the random velocity $\Delta v$ in a cloud smaller as derived from velocity - size relation for given density of the ambient medium, the gravitational instability is faster than random motions and the shells in the cloud start to produce fragments out of CPS layers. On the other hand, when the random motions inside of the cloud are larger that corresponding to the velocity - size relation, the gravitational instability is too slow and the cavities are refilled before the gravity sets in.

We compare this theoretical velocity - size relation for triggering in the CPS with the observed relation as given by Larson (1981):

$$
\Delta v(\mathrm{~km} / \mathrm{s})=1.1 \Delta r(\mathrm{pc})^{0.38} .
$$

It is interesting that starting from low $n=10 \mathrm{~cm}^{-3}$ and sizes $\Delta r=100 \mathrm{pc}$ up to the high $n=10^{6} \mathrm{~cm}^{-3}$ and sizes $\Delta r=0.01 \mathrm{pc}$ the Larson relation is always just at the theoretical velocity - size relation for triggering. We see only a slightly more gravity dominance for high densities. It shows that in average the clouds are only marginally unstable to gravitational triggering with shells, to almost the same degree on all observed scales. It implies a self-regulation of the level of turbulent random motions: the shell instability increases the level of turbulence leading to faster destruction of shells due to random motions, which decreases the triggering and new star formation.

\section{Conclusions}

The ISM is described as a bubble bath composed of moving shells expanding inside of clouds. The cold and thin CPS layer, see Elmegreen \& Lada (1978), trigger gravitational instability producing clumps with a mass spectrum with a slope $\alpha=-2.25$. The expansion time needed for the initialisation of the instability sets the velocity - size relation of the triggering, which is compared with the observational relation derived by Larson (1981). We conclude that clouds are only marginally unstable due to the gravitational instability of shells. This is valid on all the scales from $0.01-100 \mathrm{pc}$, there is only a slight increase of triggering at high densities. It also shows that there is a self-regulation between the triggering with shells and turbulent motions inside of clouds. 


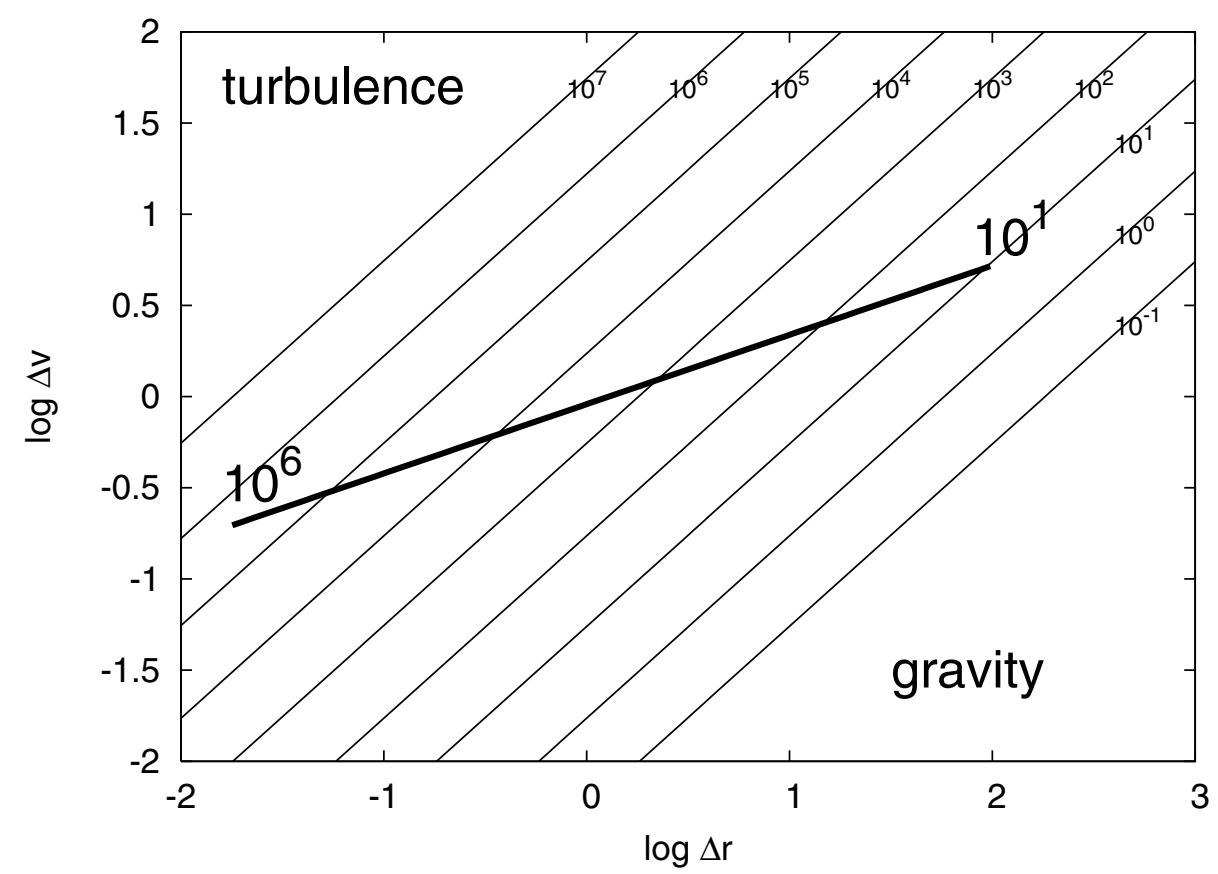

Figure 1. The velocity - size relation for the shells triggering the gravitational instability. The numbers give the density of the medium, we assume $c_{s h}=1 \mathrm{~km} / \mathrm{s}$. The thick line shows the observational Larson (1981) velocity - size relation.

\section{Acknowledgements}

I would like to acknowledge the Institutional Research Plan AV10030501 of the Astronomical Institute, Academy of Sciences of the Czech Republic, and the project LC 06014 Center for Theoretical Astrophysics.

\section{References}

Brinks, E. 2006, this volume

Ehlerová, S. 2006, this volume

Ehlerová, J. \& Palouš, J. 2005, A\&A 437, 101

Elmegreen, B.G. \& Elmegreen, D. 1978 ApJ 220, 1051

Elmegreen, B.G. \& Lada, Ch. 1978, ApJ 214, 725

Elmegreen, B.G. \& Scalo, J. 2004, ARA\&A 42, 211

Larson, R.B. 1981, MNRAS 194, 809

Palouš, J., Ehlerová, S. \& Wünsch, R. 2003 Astrophys. and Space Sci. 284, 873

Scalo, J. \& Elmegreen, B.G. 2004, ARA\&A 42, 275

Stanimirovic, S. 2006, this volume

\section{Discussion}

E. VAZQUEZ-SEMADEnI: There is only a small preference for triggering at high compared to small densities. In your comparison of the free-fall time versus the turbulent time, you conclude gravity dominates at high densities. What do you assume to associate a turbulent crossing time to a given density? This seems to implicitly assume a density size relation. 
J. PAlouš: Yes, there is a slight increase in the dominance of gravity and shell fragmentation in the high density medium. But this is a rather tiny tendency, and I like to stress the opposite: the marginal stability of shells holds on all the scales. The differences between high and low density medium are very small as concerns the triggering by shells. I have in mind the average density in a region of a given size. All the substructures like bubbles and sheets are included into an average density. The numbers I have involved in the figure are taken from Larson (1981).

I. Goldman: In the case where gravity dominates - the gravitational instability would generate a turbulence with the SAME timescale as the gravitational timescale. So the two should be perhaps considered.

J. PALOuš: I agree that any mechanism driving the turbulence inserts random motions into smaller scales. Gravitational fragmentation of shells acts in the same way and increases the internal velocity in the thin sheets. I suspect that the same cooling mechanism, which is responsible for their formation is able to act in the initial phases of the gravitational fragmentation and keep the layer at low velocity dispersion.

K. Omukai: How did you convert the dispersion relation to the mass function?

J. PALOuš: A finite volume is filled with all the unstable modes of given wavelength with a spatial frequency inversely proportional to their growth-rate. This gives the number of unstable regions as a function of wavelength or as a function of mass. The dispersion relation for a theoretical process where $\omega$ does not depend on the wavenumber $k$ gives from purely geometrical reasons the mass spectrum represented with a power law exponent equal to $\alpha=-2$. In the case of bubbles and thin layers we get steeper slopes with $\alpha \leqslant-2.25$.

J. BALly: Sub-mm observations of dusty cores have found mass spectra with a slope, $N(m) m^{-\alpha}$ with $\alpha \approx 2.3$, similar to the IMF, and the slope you calculate from the gravitational instability of a shell. Can you speculate about the connection between instabilities and the IMF?

J. PAlouš: We see the turbulent, bubble dominated medium, which is out of an equilibrium. Therefore, stars form out of layers via their fragmentation. In my opinion, the dusty cores have been formed in the same process of gravitational fragmentation of thin sheets with a mass spectrum very close to stellar. This shows that all the star forming processes leading from cores to stars probably does not change the mass spectrum, or a similar fraction of the original core mass ends in the star for all the core masses. This may be due to effective cooling at the sub-mm wavelengths, which dominates the initial phases of the evolution of the self-gravitating fragment up to the instant when it is detached from the rest of the ambient medium. 